\title{
Etiological and clinico-paraclinical correlations in case of children's acute respiratory failure
}

\author{
Iulia Armean', Cristina Oana Marginean ${ }^{1,2}$, Lorena Elena Melit1,2 \\ ${ }^{1}$ Pediatrics Clinic 1 Targu Mures, Romania \\ ${ }^{2}$ University of Medicine and Pharmacy Targu Mures, Romania
}

\begin{abstract}
Introduction. Acute respiratory failure presents very high morbidity and mortality among the pediatric patients, being one of the most frequent causes of recurring to the emergency services and of hospitalization.

Material and method. We performed a transversal retrospective study, on 153 patients diagnosed with acute respiratory failure, with the age between 2 weeks and 17 years, admitted in the Pediatrics Clinic 1 of the Emergency Clinical County Hospital Tg. Mures, between January 2015 and December 2016.

Results. The ratio between genders favored the girls 1.12:1. Regarding the age, $4.57 \%$ were newborns, $32.67 \%$ were infants, $27.45 \%$ had the age between 1 and 3 years, $22.87 \%$ were preschool-aged children (4-6 years old), while $12.41 \%$ were between 7 and 17 years old. The laboratory data pointed out leukocytosis in $65.35 \%$ of the cases, neutrophilia in $70.58 \%$, while $20.91 \%$ presented lymphocytosis, and $5.22 \%$ expressed eosinophilia. The value of C-reactive protein correlated significantly statistic with the value of erythrocyte sedimentation rate $(p=0.002)$, and with the value of platelets $(p=0.01)$, but not with the level of hemoglobin $(p=0.15)$. The inflammatory biomarkers did not correlate significantly statistic with the severity of respiratory failure. The thoracic radiography pointed out pathological modifications in $75.16 \%$ of the patients, among whom $13.91 \%$ had a typical aspect of lung condensation, and $86.09 \%$ of interstitial pneumonia. In order to establish the severity of the disease, but also the patients' evolution, we calculated a prognostic score. According to this score, $10.45 \%$ had a very good prognosis, $68.62 \%$ a good prognosis and the rest of $20.93 \%$ presented a bad prognosis.

Conclusions. The diagnosis of acute respiratory failure in pediatric patients presents both a complex and a multifactorial etiology.
\end{abstract}

Keywords: acute respiratory failure, child, acute respiratory distress
Abbreviations
ARF - acute respiratory failure
$\mathrm{CO}_{2}$ - carbon dioxide
CI - confidence interval
CRP $-\mathrm{C}$ reactiv protein
ESR - Erythrocytes sedimentation rate
$\mathbf{O}_{2}$ - oxygen
$\mathbf{P a C O}_{2}$ - partial pressure of carbon dioxide in blood

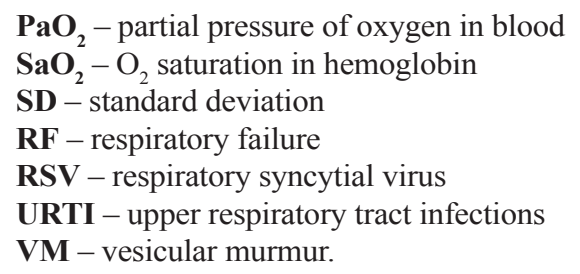

\section{INTRODUCTION}

Respiratory failure (RF) is a condition that consists in the acute or chronic inability of the respiratory system to provide the normal saturation in oxygen $\left(\mathrm{O}_{2}\right)$ of the blood and the efficient elimination of the carbon dioxide $\left(\mathrm{CO}_{2}\right)$, in order to have an adequate gases exchange for the requirements of the body metabolism (1-3). Acute respiratory failure (ARF) represents and abnormality of the blood gases, with a sudden onset, defined by a partial pressure of oxygen in blood $\left(\mathrm{PaO}_{2}\right)<60 \mathrm{mmHg}$, a partial pressure of carbon dioxide in blood $\left(\mathrm{PaCO}_{2}\right)$ $>55 \mathrm{mmHg}$, and $\mathrm{O}_{2}$ saturation in hemoglobin $\left(\mathrm{SaO}_{2}\right)$ $<90 \%$. The inappropriate blood oxygenation reduc- 
es the cellular $\mathrm{O}_{2}$ intake, inside the mitochondria, process called hypoxia and that leads to the impairment of the cellular metabolism. The decrease of $\mathrm{PaO}_{2}$, called hypoxemia, is mandatory for $\mathrm{RF}$, while the increase of $\mathrm{PaCO}_{2}$, called hypercapnia is not always present (1-2). Pediatric patients present a high susceptibility to developing disorders of the respiratory system much more severe and with a much faster evolution in comparison with adult patients $(1,3,4)$.

The commonest causes of ARF in children include upper and lower respiratory tract infections (croup, bronchiolitis, pneumonia), asthma and foreign body aspiration. We must also take into account the rare causes, such as malformations of the upper and lower respiratory tract, plastic bronchitis, pulmonary hemorrhage, acute respiratory distress syndrome and ciliary dyskinesia $(2,3)$.

The most frequent cause of cardiac instability in newborns and children is ARF. Due to this fact, the early diagnosis of ARF and the reestablishment of an efficacious ventilation and oxygenation represent the mainstay in child's resuscitation (1). Firstly, we must assess the permeability of the airways, modified breathing such as snoring or stridor, increased respiratory effort and apnea can be signs of an airways obstruction that frequently lead to ARF and cardio-respiratory arrest $(1,3)$. The signs of an increased respiratory effort include nostrils beats, stridor, intercostal, suprasternal and supraclavicular retraction. Cyanosis is a sign of severe hypoxia that indicates the urgent need for oxygen supplementation and respiratory support (1-3).

The objectives of the initial management of respiratory distress or ARF are the early stabilization of the patient's breathing and identification of the cause in order to manage correctly the afterwards therapeutic effort. The pathology of the pulmonary parenchyma includes a wide list of disorders that have in common the fact that they present the same effect on the small caliber airways and alveoli, including inflammation and exudate formation that lead to the consolidation of the pulmonary tissue with subsequent decreased of gases exchange and increase of the respiratory effort. The clinical management of these diseases includes a specific treatment such as antibiotics for bacterial pneumonia, and supportive through oxygen supplementation, non-invasive respiratory support and invasive mechanical ventilation $(1,3,5,6)$.

The aim of this study is to establish certain etiological and clinico-paraclinical correlation in patients diagnosed with ARF, to elaborate a prognostic score for these patients depending on the collected data, but also the optimization of diagnostic and treatment protocol for ARF.

\section{MATERIAL AND METHOD}

We performed a retrospective, transversal study, on a group of 153 patients diagnosed with ARF, with the age between 2 weeks and 17 years, admitted in the Pediatric Clinic 1 of the Emergency Clinical County Hospital Tg. Mures, between January 2015 and December 2017. We included all the children with clinical and paraclinical signs of ARF and also the cases with $\mathrm{SaO}_{2} \leq 95 \%$, due to the severity of the associated symptomes. The exclusion criteria consisted in incomplete anamnestic, clinical or paraclinical data. The analyzed variables were: age, gender, area of provenience, length of hospitalization, birth weight, APGAR score, actual weight, associated signs and symptoms, personal and family history, clinical exam, paraclinical data and administered treatment. These variables were interpreted according to the normal values for each age group.

For the collection of the data, we used Microsoft Office Excel package and for the statistical analysis - the SPSS soft. We used discrete quantitative variables and binary qualitative ones. For the comparison of medias/medians we used T student test with a significance threshold $95 \%$ confidence interval (CI), for the variances comparison ANOVA and chi squared tests, and Spearman coefficient for obtained correlations.

\section{RESULTS}

This study included 153 patients diagnosed with ARF based on the clinical and paraclinical data. The gender ration favored the girls 1.12:1, totalizing a number of 81 females and 72 males, among which $37.25 \%$ came from the urban area and $62.75 \%$ from the rural area.

Regarding the patients' age, we divided the children as it follows: newborns (up to 1 month of life), infants (1-12 months), small children (1-3 years of age), preschool-aged children (4-6 years of age) and school-aged children (7-17 years of age), Thus, $4.57 \%$ were newborns, infants represented $32.67 \%$, the percentage of the small children was $27.45 \%$, the preschool-aged group was $22.87 \%$ of the total, and the school-aged children represented $12.41 \%$ of the total.

Of the 153 patients included in the study, 21 $(13.73 \%)$ were born prematurely, and the rest of $132(86.37 \%)$ were born at term. 
Depending on the birth weight, newborns with the weight between 2,500 g and 3,800 g were considered eutrophic, those above $3,800 \mathrm{~g}$ overweight and those below 2,500 g, low birth weight newborn. Thus, $18.95 \%$ of the patients included in our study had low birth weight, $77.12 \%$ were eutrophic and $3.93 \%$ overweight (Fig. 1). Among the group with the birth weight below 2,500 g, 65.51\% were preterm newborns, and $34.49 \%$ were born at term. In our study the severity of ARF correlated significantly statistical with both prematurity and low birth weight ( $\mathrm{p}=0.03$, and $\mathrm{p}=0.004$, respectively).

We analyzed as risk factors for children that presented with ARF, passive smoking and home heating with biomass. Thus, among the entire group, 39 children were passive smokers, and 20 of the patients came from an environment heated with woods.

Another risk factor that can lead to ARF is represented by the pathological family and personal history regarding the respiratory tract. We realized that among the 153 patients included in our study, 53 children were formerly known with a history of respiratory disorders, and only 5 presented a relevant family history for the respiratory pathology. Of the entire study group, $15.68 \%$ presented a history of recurrent wheezing, $11.11 \%$ had a history of pneumonia, $22.87 \%$ presented repeated episodes of acute upper airways infections, and the lowest percentage, $3.70 \%$ were formerly diagnosed with asthma.

Regarding ARF etiology, in our group of study, we identified pneumonia, asthma, recurrent wheezing, bronchiolitis but also URTI. Regarding their distribution by age groups, we found that, in the case of newborns, out of a total of 7 patients, $85.71 \%$ had pneumonia and $14.29 \%$ bronchiolitis. In the infant group (majority as a number, 50) $88 \%$ experienced pneumonia, $10 \%$ bronchiolitis and $2 \%$ URTI. Of the total of 42 small children, $52.38 \%$ had pneumonia as basic pathology, $26.19 \%$ recurrent wheezing, 9.52\% bronchiolitis, $7.14 \%$ URTI and $4.77 \%$ asthma. Preschool aged-children, which represented a number of 35 patients, $62.85 \%$ were diagnosed with pneumonia, $22.85 \%$ with asthma, $8.58 \%$ with recurrent wheezing, and $5.72 \%$ with URTI. Referring to the school-aged children, the whole group of 19 patients presented pneumonia as precursor pathology towards ARF.

From the collected anamnestic data, the predominant symptom was cough in $92.15 \%$ of the cases, followed by dyspnea $(83.66 \%)$, fever (49.67\%), nasal obstruction (35.94\%), and fatigability (35.29\%). Analyzing the signs and symptoms by age group, we noticed that dyspnea was present in all the patients, followed by cough in $71.42 \%$, nasal obstruction and fever were present at $28.57 \%$ of the neonates. Similarly, in the case of infants, dyspnea was predominantly encountered $(92 \%$ of cases), followed by coughing ( $82 \%)$, fever $(50 \%)$, and nasal obstruction (40\%). Cough was the most common symptom in small children (95.23\%), dyspnea was present in $76.19 \%$ of cases, $59.52 \%$ had fever, $42.85 \%$ nasal obstruction, and 30.95\% accused fatigue. Dyspnea and cough predominant among preschool aged-children (88.57\%), 57.14 accused fatigue, $42.85 \%$ of cases were fever, and nasal obstruction was present in $20 \%$ of patients. In

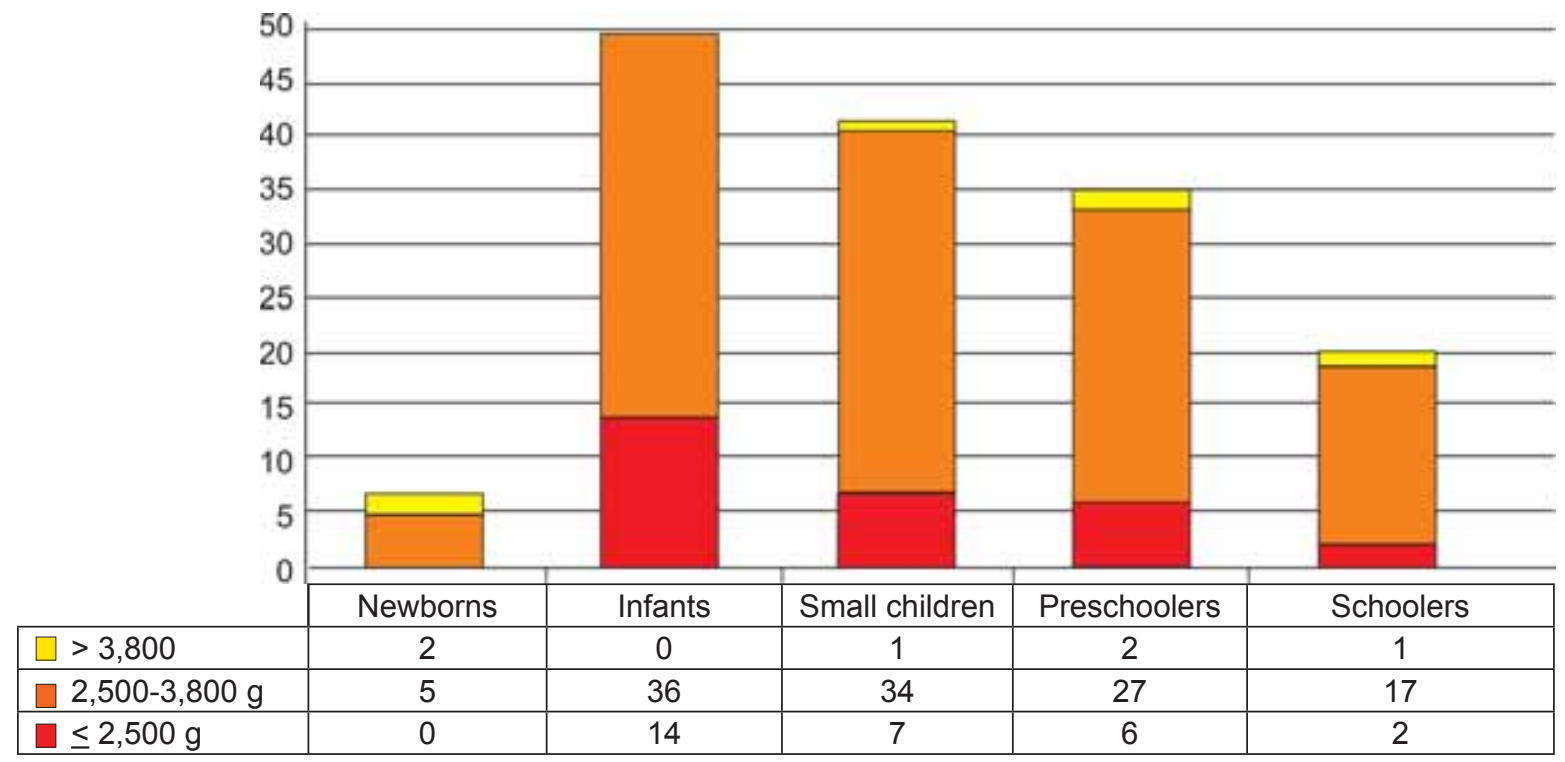

FIGURE 1. Distribution by age group and birth weight 
the case of school-aged children, ARF was predominantly manifested by cough, dyspnea and fatigue $(94.73 \%)$, fever was present in $47.36 \%$ of cases, and nasal obstruction in $42.11 \%$ of patients.

Regarding the clinical exam, we noticed that 99 of the patients presented at auscultation modifications of the vesicular murmur (VM), 28 presented diminished VM and in 26 we identified rough VM. Also, we identified sibilants in 78 patients, crackles in 49 cases, and 26 patients presented no rales at auscultation. Among the associated signs and symptoms, the retraction of the auxiliary respiratory muscles was present in 113 of the cases, wheezing was identified in 43 cases, and tachypnea, which most of the times accompanies the muscle retraction, was present in 100 of the patients diagnosed with ARF.

The laboratory parameters pointed out leukocytosis in $65.35 \%$ of the cases, neutrophilia in $70.58 \%$ of the patients, while $20.91 \%$ of the cases presented lymphocytosis, and 5.22\% expressed eosinophilia. From the total of 153 patients included in the study, $62.09 \%$ had CRP values $>0.5 \mathrm{mg} / 1$, and $37.91 \%$ had normal values (CRP $<0.5 \mathrm{mg} / \mathrm{l})$. In those who presented positive CRP, the values of this inflammatory marker were between $1.75 \mathrm{mg} / 1$ and 300 $\mathrm{mg} / \mathrm{l}$. Also, 59 of the cases included in our study associated anemia. We correlated the values of the CRP and hemoglobin, but we did not obtain a significantly statistical correlation. Contrariwise, we identified significantly statistical correlations between the values of the CRP and platelets $(p=0.01)$.

Among the 153 patients included in our study, $75.16 \%$ presented pathological modification on the thoracic radiography, but only a percentage of $13.91 \%$ were interpreted as a typical aspect of lung condensation, while $86.09 \%$ were defined as interstitial pneumonia (Fig. 2).

We also performed certain correlations between the clinical and paraclinical data.

Radiological changes considered pathological were: increased interstitial design, airspace opacity, lobar consolidation, interstitial opacities or pleurisy.

Thus, we noticed no significantly statistical correlations between the thoracic radiography and the CRP $(p=0.25)$, but it significantly correlates with the value of ESR $(p=0.02)$. We also identified a statistically significant correlation between the inflammatory markers analyzed in our study, CPR and ESR $(p=0.002)$

Among the study group, $87.58 \%$ of the cases benefited by antibiotic treatment, $84.96 \%$ of the patients required steroids, $95.42 \%$ received symptomatic treatment and $98.03 \%$ benefited by a bronchodilator. We mention that all the patients included in our study received adjuvant therapy with $\mathrm{O}_{2}$.

In order to establish the severity of the pathology, but also the prognosis of the patients admitted with ARF, we calculated a prognostic score. We included the personal history of airways infections, the birth at term or prematurely, the APGAR score $<8$, fever, the values of CRP, ESR and leukocytes, the saturation of hemoglobin in $\mathrm{O}_{2}$ and the aspect of the thoracic radiography. Each of these criteria brought an additional point in case of positivity: the presence of a personal history of respiratory infections, prematurity, APGAR $<8$, fever $>38.5^{\circ} \mathrm{C}$,

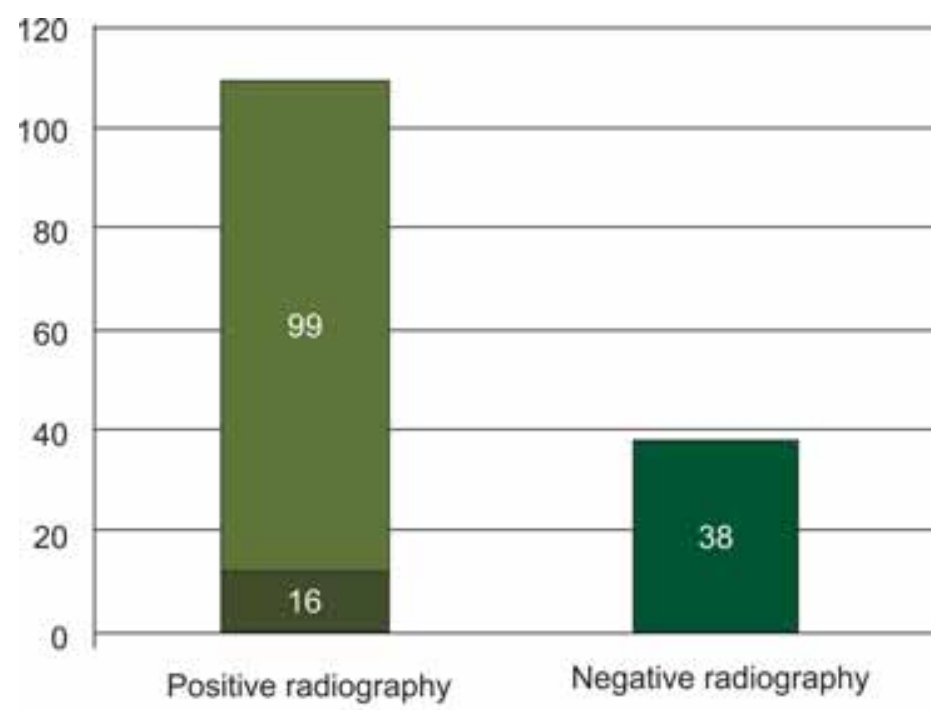

E Interstitial pneumoniae

Tipical pneumoniae 
presence of dyspnea, positive CPR, positive ESR, leukocytes over normal group age, $\mathrm{SaO}_{2}<92 \%$, and the presence of pathological changes on chest radiographs.

A prognostic score below or equal to 3 classified the patient in the group of those with very good prognosis, a score between 4 and 6 inclusively defined the patients as having a good prognosis, while a score between 7 and 10 included the patients in the group with bad prognosis. As a result of this score, 16 of the patients included in our study had a very good prognosis, 105 were defined as having a good prognosis, while the rest of 32 presented a bad prognosis. The medium number of hospitalization days was $7.26 \pm 9.29 \mathrm{DS}$. We correlated the prognostic score with the length of hospitalization, but we did not obtain statistical significance $(\mathrm{p}=0.52)$ (Spearman correlation coefficient $\mathrm{r}=0.51)$, but it significantly statistical correlated with the severity of cardiac failure $(\mathrm{p}=0.0008)$.

We also mention that in our study there were no deaths recorded.

\section{DISCUSSIONS}

One of the problems encountered in the early and correct diagnosis of ARF is the symptomatology described by the care-givers who frequently tend to overestimate it misleading the physician in many cases. Even though they should guide the children's triage and afterwards management, in many cases they hinder the establishment of an early diagnosis. In our study, we noticed that the major symptoms which guided the patients to the emergency room were cough, followed by dyspnea, fever, nasal obstruction and fatigability. In a study performed on 535 patients diagnosed with different degrees of respiratory failure, the authors analyzed the symptoms presented by the patients, and they underlined the fact that cough had moderate sensibility and reduced specificity, while dyspnea, wheezing and dehydration had high specificity. The same study showed that $\mathrm{SaO}_{2}<94 \%$ and fever $>39^{\circ} \mathrm{C}$ were the most specific and reliable signs and symptoms that could guide the correct diagnosis of respiratory failure (5). Every patient included in our study benefited by a monitoring of all the vital signs during the admission, among which also the respiratory frequency, but according to the specialty literature, this parameter is lacked of accuracy and possibly influenced by the length of monitoring, the obtained value being more precise in case of increasing the interval of counting. These data are sustained by recent studies which state the fact that respiratory frequency in not a predictable and useful parameter in diagnosing ARF $(8,9)$.

As diagnostic criteria for ARF of the patients included in our study, we used the value of $\mathrm{SaO}_{2}$. Thus, we noticed that most of the patients $(81.69 \%)$ had saturations below $92 \%$. Nevertheless, all the patients included in our study were diagnosed with ARF based on the accumulation of the clinical and paraclinical data that pointed out the severity of the patients' condition. Recent studies sustain the fact that the definition of ARF supposes a $\mathrm{SaO}_{2}$ below $92 \%(9)$.

In our study, we also analyzed the aspect of the thoracic radiography, which suggested in most of the cases modifications suggesting an interstitial pneumonia that has generally a viral etiology. A recent study realized on a group of 733 patients diagnosed with pneumonia pointed out that more than $50 \%$ of the cases had a viral etiology, especially respiratory syncytial virus (RSV) (10). Another study performed in Brazil on a group of children with the age below 5 years diagnosed with viral pneumonia showed that RSV was responsible for $37.3 \%$ of the cases (11). Similarly, this hypothesis is sustained also by another study which proved the fact that RSV predominates significantly in comparison to other viruses $(100: 14)$ or bacteria (100:15), underlining therefore its important role in severe lower airways infections in case of infants and small children (12). Also, it is important to mention that viral respiratory infections are by far the most frequent among the children worldwide (13).

In the specialty literature is described the fact that, besides the viral etiology, many times acellular bacteria cause atypical pneumonias that can mimic radiologically the aspect of viral pneumonia. Recent studies prove the fact that among the pediatric patients increased significantly the number of pneumonias causes by atypical bacteria such as Chlamydia pneumoniae or Mycoplasma pneumoniae (14). Therefore, we cannot exclude the possibility that a part of the pneumonias from our study in which the radiological aspect showed interstitial impairment, had as a possible etiology these types of bacteria. Multiple studies showed that most of the pneumonias caused by Mycoplasma pneumoniae expressed wheezing, fever and cough (15-17), which were also encountered in our study. Another study which compared the signs and symptoms encountered in pneumonia caused by Mycoplasma pneumoniae and those caused by other common bacteria found in this pathology, underlined a higher frequency of headache, cough and wheezing in 
case of patients with pneumonia caused by this atypical bacterium (18). In addition, another study stated that these signs can even guide the diagnosis (19).

Most of the patients included in our study benefited by a therapeutic regimen that consisted in an association between antibiotics, steroids, bronchodilators and symptomatic drugs. The association of steroids in infectious respiratory pathology is controversial, but recent studies sustain the fact that they reduce the mortality and hospitalization length of respiratory tract infections that can lead in many cases, without a proper treatment, to $\operatorname{ARF}(20,21)$.

\section{CONCLUSIONS}

The diagnosis of ARF in pediatric patients presents both a complex and multifactorial determin- ism, but also a wide etiology. Our study pointed out an increased prevalence of ARF among females, especially from rural area, affecting most frequently infants and small children. Even though low birth weight is a risk factor for ARF, our study underlines the fact that this condition is frequently encountered among eutrophic patients, with normal birth weight. Thoracic radiography suggests in most of the cases included in the study a viral etiology or probably due to atypical bacteria. Even though according to the prognostic score, a significant percentage presented a bad prognosis, it does not influence significantly statistic the length of hospitalization, but it correlates significantly with the severity of ARF.

The patients' evolution and prognosis depends on both external factors and the patient's individual susceptibility.

Conflict of interest: none declared Financial support: none declared

\section{REFERENCES}

1. Robert M. Kliegman, Bonita F. Stanton, Joseph W. St. Geme et al. Nelson Textbook of Pediatrics. 20th ed. Philadelphia: Elsevier; 2016.

2. Schneider J., Sweberg T. Acute respiratory failure. Critical Care Clinic. 2013; 29:167-183.

3. Hammer Jung. Acute respiratory failure in children. Paediatric Respiratory Reviews. 2013; 14:64-69

4. Rubarth L.B.Q.J. Respiratory developemnt and Respiratory Distress Syndrom. Neonatal Network. 2015; 34:231-238.

5. Blacklock C., Mayon White R., Coad N., Thompson M. Which symptoms and clinical features correctly identify serious respiratory infection in children attending a paediatric assessment unit? Archives of Disease in Childhood. 2011; 96:708-714.

6. Moretti C., Papoff P., Caresta E. et al. Management of acute injury and acute respiratory distress syndrome in infants. Early Human Developement. 2013; 89:96-98.

7. Cardoso M.R., Nascimento-Carvalho C.M., Ferrero F. et al. Adding fever to WHO criteria for diagnosing pneumonia enhances the ability to identify pneumonia cases among wheezing children. Arch Dis Child. 2011; 96:58-61.

8. Hay A.D., Wilson A., Fahey T., Peters T.J. The inter-observed agreement of examining pre-school children with acute cough $-\mathrm{a}$ nested study. BMC Fam Pract. 2004; 5:4-5.

9. Hammer J. Acute respiratory failure in children. Pediatric Respiratory Reviews. 2013; 14:64-69.

10. El Bacha N., El Rifai N., Diaz I., El Kholy A. Contribution of viruses to severe pneumonia in children. Egyptian Pediatric Association Gazette. 2013; 61:73-77

11. Beserra P.G., Britto M.C., Correia J.B. et al. Viral and atypical bacterial detection in acute respiratory infection in children under five years. PLos One. 2011; 6:4.

12. Vieira S.E., Stewien K., Queiroz A.O. Clinical patterns and seasonal trends in respiratory syncytial virus hospitalization in Sao Paulo, Brazil. Rev Inst Med Trop Sao Paulo. 2001; 43:125-131.

13. Sinaniotis C.A., Sinaniotis A.C. Community-acquired pneumonia in children. Curr Opin Med. 2005; 11:218-25.

14. Biljana Medjo, Marina Atanaskovic-Markovic et al. Mycoplasma pneumoniae as a causative agent of community-acquired pneumonia in children: Clinical features and laboratory diagnosis. Italian Journal of Pediatrics. 2014; 40:104-111.

15. Defilippi A. Epidemiology and clinical features of Mycoplasma pneumoniae infection in children. Respir Med. 2008; 2:1762-1768.

16. Almasri M., Diza E., Papa A. Mycoplasma pneumoniae respiratory tract infections among Greek children. Hippokratia. 2011;15:147-152.

17. Samransamruajkit R., Siriwan J., Witan W. Prevalence of mycoplasma and Chlamydia pneumoniae in severe comunity-acquired pneumonia among hospitalized children in Thailand. JPN J Infect Dis. 2008; 61:36-39.

18. Sidal M., Kilic A., Unuvar E. et al. Frequency of Chlamydia pneumoniae and Mycoplasma pneumoniae infections in children. J Trop Pediat. 2007; 53:225-231.

19. Vervloet L.A., Camargos M.P.A., Soares F.R.D. et al. Clinical, radiographic and hematological characteristics of Mycoplasma pneumoniae pneumonia. J Pediatr. 2010; 86:480-487.

20. Wei-Fang Wu, Qiang Fang, Guo-Jun H.E. Efficacy of corticosteroid treatment for severe community-acquired pneumonia. American Journal Of Emergency Medicine. 2017.

21. Ricardo M. Fernandez, Marta Oleszczuk, Charles R. Woods et al. The Cochrane Library and safety systemic corticosteroids for acute respiratory conditions in children. An overview of reviews. EvidenceBased Child Health. 2014; 9:733-747. 